\title{
TILLANDSIA BOREALIS (BROMELIACEAE), UNA NUEVA ESPECIE DEL NORTE DE MÉXICO
}

\author{
Ana Rosa LóPEZ-FerRARI y AdOLFO ESPEJO-SERNA ${ }^{1}$ \\ Herbario Metropolitano, Departamento de Biología, Div. C.B.S., \\ Universidad Autónoma Metropolitana-Iztapalapa, Apdo. Postal 55-535, \\ Iztapalapa 09340, México, D.F., México. \\ 'Autor para la correspondencia. Correo-e: aes@xanum.uam.mx
}

\begin{abstract}
Resumen: Se describe e ilustra Tillandsia borealis, conocida de los estados de Durango y Sinaloa, México. Los caracteres que permiten reconocer a la especie aquí propuesta son: las inflorescencias erectas, las espigas elípticas a ovado elípticas, provistas de un pedúnculo corto, robusto y ebracteado y los pétalos verdes pálidos. Se compara T. borealis con T. carloshankii Matuda, T. prodigiosa Baker y T. macrochlamys Baker las cuales presentan características similares y se incluye un cuadro comparativo y un mapa de distribución de las mismas.

Palabras clave: Bromeliaceae, Durango, México, Sinaloa, Tillandsia.

Abstract: Tillandsia borealis known from the states of Durango and Sinaloa, Mexico, is described and illustrated. The new species here proposed is recognized by its erect inflorescences, its elliptic to ovate elliptic spikes with short and robust peduncles, and its pale green petals. T. borealis is compared with T. carloshankii Matuda, T. prodigiosa Baker, and with T. macrochlamys Baker, species with similar characteristics, and a comparative table and a distribution map of these taxa are included.

Key words: Bromeliaceae, Durango, Mexico, Sinaloa, Tillandsia.
\end{abstract}

C on poco más de 220 representantes en México (Espejo-Serna et al., 2004, 2007; Ehlers, 2005, 2006a, 2006b, 2006c, 2006d; Espejo-Serna y LópezFe rrari, 2005; López-Ferra ri et al., 2006), de las cuales $181(82 \%)$ son epífitas, Tillandsia es, entre las monocotiledóneas mexicanas, el género con mayor número de taxa. A pesar de lo dicho, existen aún taxa por describir, ya que exploraciones en varios estados del país, así como revisiones de especímenes en diversos herbarios, encaminadas a obtener el inventario de la fl ora bromeliológica mexicana, nos permitieron descubrir un nuevo taxon del género que aquí proponemos como:

Tillandsia borealis López-Ferrari et Espejo-Serna sp. nov. Figuras 1d y 2a-c.

Herbae epiphyticae, acaules, rosulatae, florens usque ad $70 \mathrm{~cm}$ altae. Folia basalia, vaginae distinctae, ellipticae,
$12-16 \mathrm{~cm}$ longa e, $8-9 \mathrm{~cm}$ latae; laminae anguste triangulaii-lanceolatae, $35-55 \mathrm{~cm}$ longa e, $2-3 \mathrm{~cm}$ latae, erectae, apice recurvatae; scapus infl o rescentiae erectus, teres, 10 $20 \mathrm{~cm}$ longus; infl o rescentia bipartita; spicae 16-30, ellipticae vel ovato-ellipticae, fo rtiter complanat ae, $4-6 \mathrm{~cm}$ longa e, 1.8-2.3 cm latae, pedunculate; pedunculi robustes 1$1.6 \mathrm{~cm}$ longi; bracteae fl orales distichae, imbric atae, triangulares vel ovato-triangulares, 2-3.5 cm longa e, 0.8-1.8 $\mathrm{cm}$ latae, carinatae, virides, nervatae vel levis; petala oblongo-spathulatae, 5-5.9 cm longa e, 6-8 mm latae, pallide viridia; stamina et stylus ex serta.

TIPO: México, Sinaloa. Municipio de Concordia, ca. $3 \mathrm{~km}$ después de El Palmito, rumbo a Concordia, carretera Durango-Mazatlán, $23^{\circ} 33^{\prime} 40^{\prime \prime} \mathrm{N}, 105^{\circ} 51^{\prime} 03^{\prime}$ ' O, alt. 1,971 m s.n.m., bosque de pino-encino, 26 julio 2006, A. EspejoSerna, A.R. López-Ferrari, J. Ceja y A. Mendoza-R. 6573 (Holotipo: UAMIZ; Isotipos: IEB, MO). 
Hierbas epífitas, acaules, de 60 a $70 \mathrm{~cm}$ de alto incluyendo la inflorescencia, amosetadas, la roseta obcónica, de 35 a $45 \mathrm{~cm}$ de diámetro. Hojas numerosas, vainas pardas claras, discoloras con respecto a la lámina, opacas, elípticas, de 12 a $16 \mathrm{~cm}$ de largo, de 8 a $9 \mathrm{~cm}$ de ancho, densamente punctulado lepidotas en ambas superficies. Láminas verdes, angostamente triangular-lanceoladas, e rectas en la base, recurvadas en el ápice, de 35 a $55 \mathrm{~cm}$ de largo, de 2 a $3 \mathrm{~cm}$ de ancho en la base, atenuadas, enteras, filiformes hacia el ápice, densamente punctulado lepidotas en ambas superficies, ate rciopeladas al tacto. Escapo rollizo, de 10 a $20 \mathrm{~cm}$ de largo, de 5 a $6 \mathrm{~mm}$ de diámetro, inconspicuo y más corto que las hojas, erecto. Brácteas del escapo foliáceas, imbricadas y cubriéndolo totalmente, disminuyendo su tamaño hacia la parte distal, de 20 a $35 \mathrm{~cm}$ de largo. Inflorescencia erecta, dos veces dividida, con 16 a 30 espigas divaricadas a adpresas, el raquis conspicuamente acostillado cuando seco. Brácteas primarias ve rdes a rosadas, ovado-triangulares a ovado-lanceoladas, de 4.5 a $35 \mathrm{~cm}$ de largo, de 1.5 a $3.5 \mathrm{~cm}$ de ancho, las inferi o res foliáceas, la lámina largamente triangular, atenuada, las superi ores bracteosas, 1 a rgamente at enudas a acuminadas en el ápice. Espigas elípticas a ovado-elípticas, de 4 a $6 \mathrm{~cm}$ de largo, de 1.8 a $2.3 \mathrm{~cm}$ de ancho, fuertemente comprimidas, con 4 a 6 flores, pedunculadas, los pedúnculos cortos, robustos, ebracteados, de 1 a $1.6 \mathrm{~cm}$ de largo. Brácteas florales ve rdes a ve rdes blanquecinas con el ápice rosado, triangulares a ovado triangulares, de 2 a 3.5 cm de largo, de 0.8 a $1.8 \mathrm{~cm}$ de ancho cuando aplanadas, fuertemente carinadas a bicarinadas, nervadas a lisas cuando secas, agudas a acuminadas, glabras a lige ramente lepidotas en el ápice. Sépalos ve rdes a ve rdes blanquecinos, libres, angostamente elípticos a lanceolados, de 2 a $2.7 \mathrm{~cm}$ de largo, de 5 a $9 \mathrm{~mm}$ de ancho, glabros, enteros, agudos a acuminados en el ápice, los dos posterio res carinados. Pétalos oblongo-espatulados, de 5 a $5.9 \mathrm{~cm}$ de largo, de 6 a $8 \mathrm{~mm}$ de ancho, refl exos en el ápice, ve rdes pálidos en su mitad apical, blancos en su mitad basal. Estambres ex sertos, desiguales, filamentos lineares, aplanados en la porción apical, de 5.3 a $6.5 \mathrm{~cm}$ de largo, blancos en la base, ve rdes pálidos en el ápice. Anteras amarillas, basifijas a subbasifijas, oblongas, de $c a .3 \mathrm{~mm}$ de largo. O va rio ovo ide, de 6 a $9 \mathrm{~mm}$ de largo, de 2 a $4 \mathrm{~mm}$ de diámetro, verde. Estilo exserto, linear, verde-blanquecino, de 5.4 a $6.4 \mathrm{~cm}$ de largo. Estigma blanco a verde, conduplicado-espiral (tipo II sensu B rown y Gilmartin, 1984). Cápsula no vista.

Paratipos. MÉXICO, Durango: Municipio Pueblo Nuevo, El Salto, Durango to Mazatlán, col. II-III.1969, fl. VII.1969, C. Geriche s. n. (US 2571285A); E of La Palmita on Mazatlán - Dura n go ro ad, 2,003 m, H.E. Luther et al. 2954 (SEL 68205); $3 \mathrm{~km} \mathrm{E}$ of La Palmita on Mazatlán - Durango road, pine-oak forest, 2,035 m, H.E. Luther et al. 2955 (SEL 68204); along highway betwe en Llano Grande and Las Rusias, 2 miles by road southsouthwest of C. Durango, Pine forest, J.H. Maysilles

Cuadro 1. Caracteres comparativos entre Tillandsia borealis y especies afines.

\begin{tabular}{|c|c|c|c|c|}
\hline Caracter & T. borealis & T. carloshankii & T. macrochlamys & T. prodigiosa \\
\hline Vainas foliares & $\begin{array}{l}\text { elípticas, pardas } \\
\text { claras, opacas }\end{array}$ & $\begin{array}{l}\text { largamente ovadas, } \\
\text { pardas oscuras, } \\
\text { lustrosas }\end{array}$ & $\begin{array}{l}\text { elípticas, pardas } \\
\text { claras, opacas }\end{array}$ & $\begin{array}{l}\text { oblongo-elípticas, } \\
\text { pardas claras, opacas }\end{array}$ \\
\hline Láminas foliares & $2-3 \mathrm{~cm}$ de ancho & $\begin{array}{l}\text { hasta de } 1.5 \mathrm{~cm} \\
\text { de ancho }\end{array}$ & 1.5-2.7 cm de ancho & $3.7-5 \mathrm{~cm}$ de ancho \\
\hline Inflorescencia & erecta & erecta & péndula & péndula \\
\hline Brácteas primarias & verdes a rosadas & verdes a escarlatas & rojas & verdes a rosadas \\
\hline Espigas & $\begin{array}{l}\text { pedúnculo de } 1 \text { a } \\
1.6 \mathrm{~cm} \text { de largo }\end{array}$ & $\begin{array}{l}\text { pedúnculo de } \\
\text { ca. } 6 \mathrm{~mm} \text { de largo }\end{array}$ & $\begin{array}{l}\text { pedúnculo ausente } \\
\text { o de menos de } \\
3 \mathrm{~mm} \text { de largo }\end{array}$ & $\begin{array}{l}\text { pedúnculo de } \\
0.6-2 \mathrm{~cm} \text { de largo }\end{array}$ \\
\hline Brácteas florales & $\begin{array}{l}\text { triangulares a } \\
\text { ovado-triangulares, } \\
2-3.5 \times 0.8-1.8 \mathrm{~cm} \text {, } \\
\text { glabras a ligeramente } \\
\text { lepidotas en el ápice }\end{array}$ & $\begin{array}{l}\text { oblongo-triangulares, } \\
2.5 \times 1.2 \mathrm{~cm} \text {, } \\
\text { densamente lepidotas }\end{array}$ & $\begin{array}{l}\text { oblongo-elípticas, } \\
2.2-2.8 \times 0.8-1.4 \mathrm{~cm}, \\
\text { glabras }\end{array}$ & $\begin{array}{l}\text { ovadas a triangulares, } \\
2.5-3.5 \times 1.4-16 \mathrm{~cm} \text {, } \\
\text { glabras }\end{array}$ \\
\hline Pétalos & $\begin{array}{l}\text { verdes pálidos, } \\
5.1-6 \mathrm{~cm} \text { de largo }\end{array}$ & $\begin{array}{l}\text { verdes pálidos, } \\
5.2-5.7 \mathrm{~cm} \text { de largo }\end{array}$ & $\begin{array}{l}\text { violetas, } 4.9-5.1 \mathrm{~cm} \\
\text { de largo }\end{array}$ & $\begin{array}{l}\text { verdes pálidos, } \\
5.6-5.7 \mathrm{~cm} \text { de largo }\end{array}$ \\
\hline Distribución & Durango y Sinaloa & Oaxaca & Veracruz & $\begin{array}{l}\text { Colima, Guerrero, } \\
\text { Jalisco, Edo. de México, } \\
\text { Michoacán, Morelos, } \\
\text { Oaxaca y Puebla }\end{array}$ \\
\hline
\end{tabular}


7089A (MICH, US 2088639); El Amole "La Mole", 1,8002,100 m, bosque de pino-encino, P. Tenorio-L., C. Romero de T. y J. Ignacio-S. 9741 (UAMIZ). Sinaloa: Municipio de Concordia, $48 \mathrm{~km}$ from Villa Unión turn-off to Durango, open oak, some pine, 1,700 m, 10-08-1967, A.J. Gilmartin 1301 (SEL 62641, US 3160062); $40 \mathrm{~km}$ from Villa Unión tum-off to Durango, open oak, some pine, 1,700 m, 10-08-1967, A.J. Gilmartin 1304 (SEL 62644); Mile 53; Villa Unión turn - off to Durango, pine-oak forest, 1,850 m s.n.m., 27-08-1968, A.J. Gilmartin 1441 (SEL 62647); Mile 51; Villa Unión turn-off to Durango, pineoak forest, 1,850 m s.n.m., 27-08-1968, A.J. Gilmartin 1442 (SEL 62642), 1446 (SEL 62643); ca. $50 \mathrm{~km}$ Mazatlán - Duran go road, 10-03-1994, P. Koide and W. Schuster s. n. (SEL 71183); carre te ra Durango-Mazatlán, bosque de Pinus-Quercus, 2,050 m s.n.m., 5-01-1980, $R$. Vega-A., J.S. Palazuelos-N. y J.M. Aguilar-P. 742 (FCME 10402).

Distribución y ecología. Tillandsia borealis se conoce hasta ahora sólo de los estados de Durango y Sinaloa (figura 3), en los municipios de Pueblo Nuevo y Concordia respectivamente, donde crece como epífita sobre Pinus y/o Quercus en bosques de pino-encino, en un intervalo altitudinal que va de los 1,700 a los 2,000 m s.n.m. Florece de julio a agosto.

Parte del material herborizado hasta ahora conocido del nuevo taxon aquí propuesto fue inicialmente determinado con el nombre de Tillandsia prodigiosa Baker (figuras 1c, 3), seguramente debido al color verde de los pétalos y a las largas inflorescencias. Posteriormente, algunos ejemplares fueron identificados como T. macrochlamys Baker (figuras $1 b, 3$ ), debido quizás a la presencia de las brácteas primarias amplias que cubren a las espigas cortas que presentan un robusto pedúnculo. Sin embargo, pensamos que la especie más parecida a $T$. borealis es en realidad $T$. carloshan kii Matuda (figuras 1a, 3), taxon endémico del estado de Oaxaca. En el cuadro 1 comparamos algunas de las características de los taxa arriba mencionados, las cuales permiten distinguirlos claramente. Los caracteres que permiten reconocer a la especie aquí propuesta son: las inflorescencias erectas, las espigas elípticas a ovado elípticas, provistas de un pedúnculo corto, robusto y ebracteado y los pétalos verdes pálidos.

El epíteto específico hace referencia a la distribución norteña de la especie en México.

Tillandsia borealis se ubica en el subgénero Tillandsia de la clasificación propuesta por Mez (1896) y seguida por Smith y Downs (1977) en su monografía de la familia. Asimismo, pertenece al subgrupo 3 del grupo 1 propuesto por Gardner (1986) en su trabajo sobre la clasificación preliminar del género Tillandsia. Cabe señalar, sin embargo, que recientes publicaciones relativas a la taxonomía de la subfamilia Tillandsioideae (Spencer, 1993; Grant, 1995a, 1995b; Terry et al., 1997; Espejo-Serna, 2002; Barfuss et al., 2004, 2005; Till y Barfuss, 2006), han puesto de manifiesto que dichas clasificaciones son artificiales y han sugerido la necesidad de llevar a cabo una revisión cuidadosa de la delimitación genérica e infragenérica en la subfamilia.

\section{Agradecimientos}

Agradecemos a Javier García-Cruz, a Walter Till y a Jorge Meave la revisión crítica del manuscrito. Un especial reconocimiento a Jacqueline Ceja y Aniceto Mendoza por su incondicional e invaluable apoyo en el trabajo de campo. Los curadores de los herbarios AA, CHAP, CICY, CIIDIR, CODAGEM, ENCB, F, FCME, GH, IBUG, IEB, K, MEXU, MICH, MO, P, SEL, TEX, UAMIZ, US, WU y XAL (Holmgren et al., 1990) nos dieron todas las facilidades para consultar sus colecciones. Parte de este trabajo pudo llevarse a cabo gracias al apoyo de la beca Elizabeth Bascom otorgada en el año 2004 por el Missouri Botanical Garden a A. R. López-Ferrari.

\section{Literatura citada}

Barfuss M.H.J., Samuel M.R. y Till W. 2004. Molecular phylogeny in subfamily Tillandsioideae (Bromeliaceae) based on six cpDNA markers: An update. Journal of the Bromeliad Society 54:9-17.

Barfuss M.H.J., Samuel M.R., Till W. y Stuessy T.F. 2005. Phylogenetic relationships in subfamily Tillandsioideae (Bromeliaceae) based on DNA sequence data from seven plastid regions. American Journal of Botany 92:337-351.

Brown G.K. y Gilmartin A.J. 1984. Stigma structure and variation in Bromeliaceae-neglected taxonomic characters. Brittonia 36:364-374.

Ehlers R. 2005. Tillandsia zacualpanensis Ehlers \& Wülfinghoff spec. nov. Die Bromelie 2005:12-16.

Ehlers R. 2006a. Tillandsia huamelulaensis, a new species. Journal of the Bromeliad Society 56:56-59.

Ehlers R. 2006b. Tillandsia nicolasensis, a new species. Journal of the Bromeliad Society 56:70-72.

Ehlers R. 2006c. Tillandsia comitanensis, a new highland species from Chiapas, Mexico. Journal of the Bromeliad Society 56:116-119.

Ehlers R. 2006d. Tillandsia celata Ehlers \& Lautner, spec. nov. Die Bromelie 2006:8-12.

Espejo-Serna A. 2002. Viridantha, un género nuevo de Bromeliaceae (Tillandsioideae) endémico de México. Acta Botanica Mexicana 60:25-35.

Espejo-Serna A. y López-Ferrari A.R. 2005. Una nueva especie de Tillandsia (Bromeliaceae) del Occidente de México. Acta Botanica Mexicana 72:53-64.

Espejo-Serna A., López-Ferrari A.R., Ramírez-Morillo I., Holst B.K., Luther H.E. y Till W. 2004. Checklist of Mexican Bromeliaceae with notes on species distribution and levels of endemism. Selbyana 25:33-86.

Espejo-Serna A., López-Ferrari A.R. y Till W. 2007. Tillandsia suesilliae Espejo-Serna, López-Ferrari et W. Till, a new species 

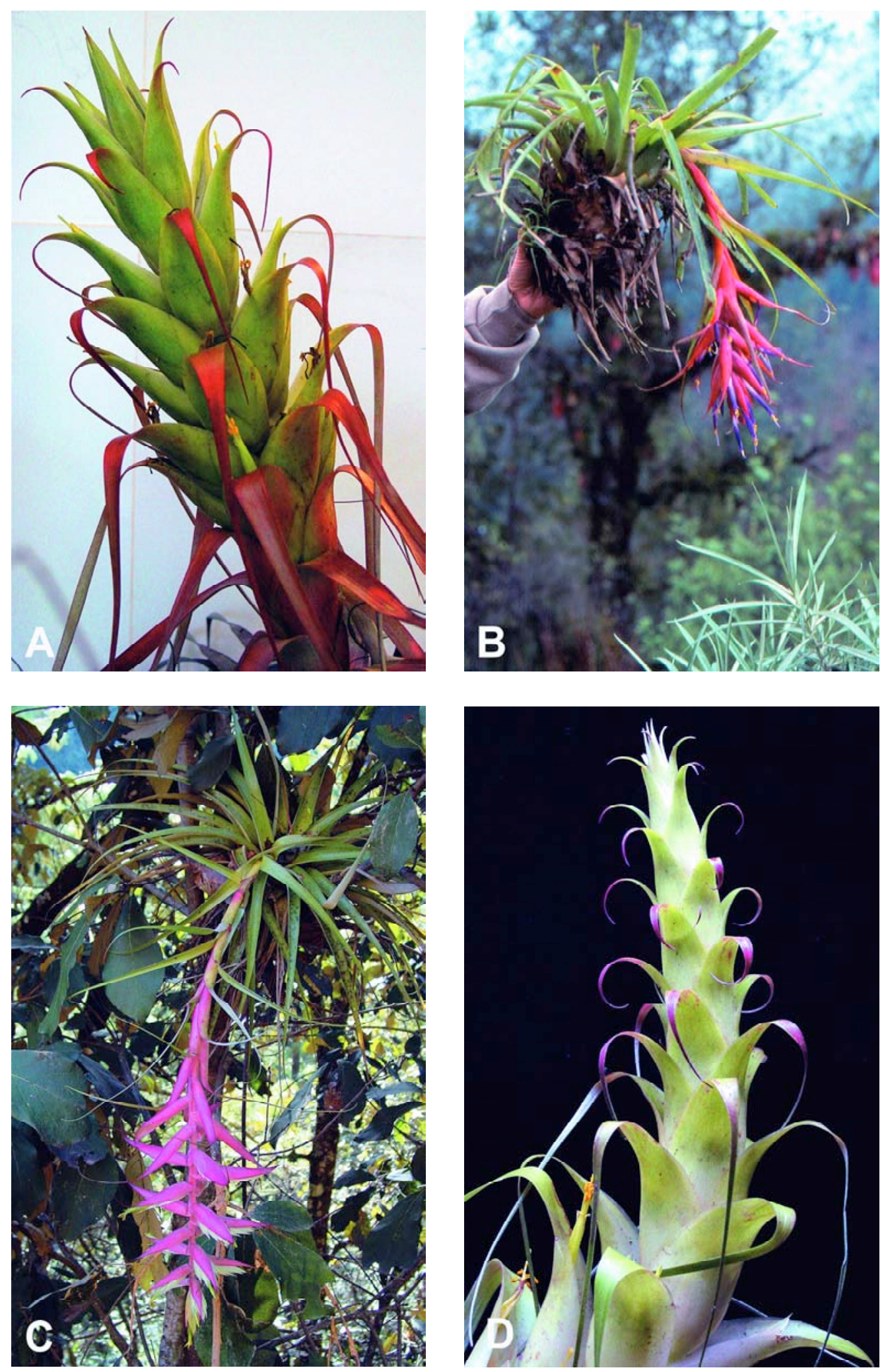

Figura 1. A. Tillandsia carloshankii Matuda. B. Tillandsia macrochlamys Baker. C. Tillandsia prodigiosa (Lem.) Baker. D. Tillandsia borealis López-Ferrari y Espejo-Serna. 

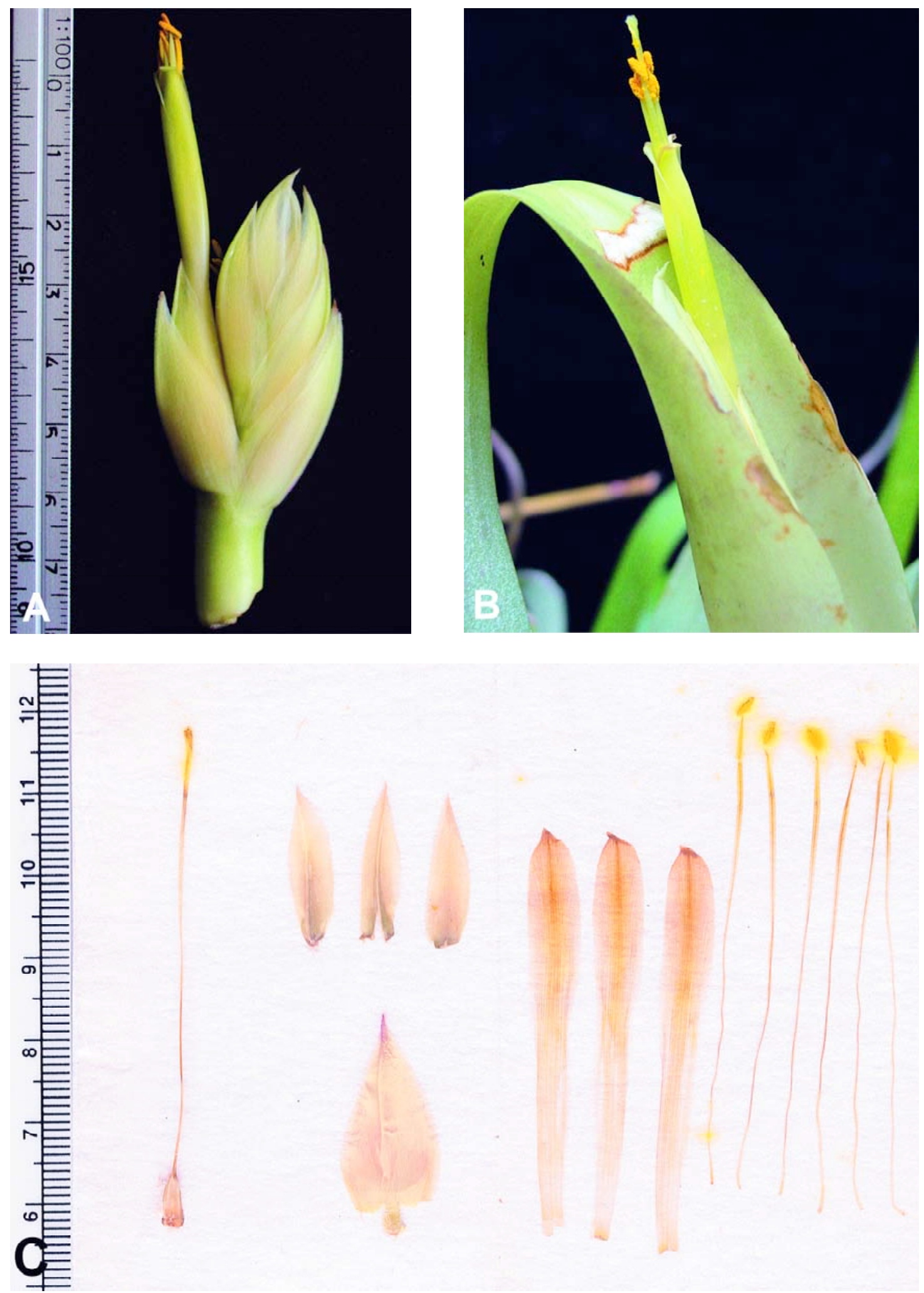

Figura 2. Tillandsia borealis López-Ferrari y Espejo-Serna. A. Espiga. B. Detalle de la inflorescencia mostrando la bráctea primaria. C. Flor disecada. 


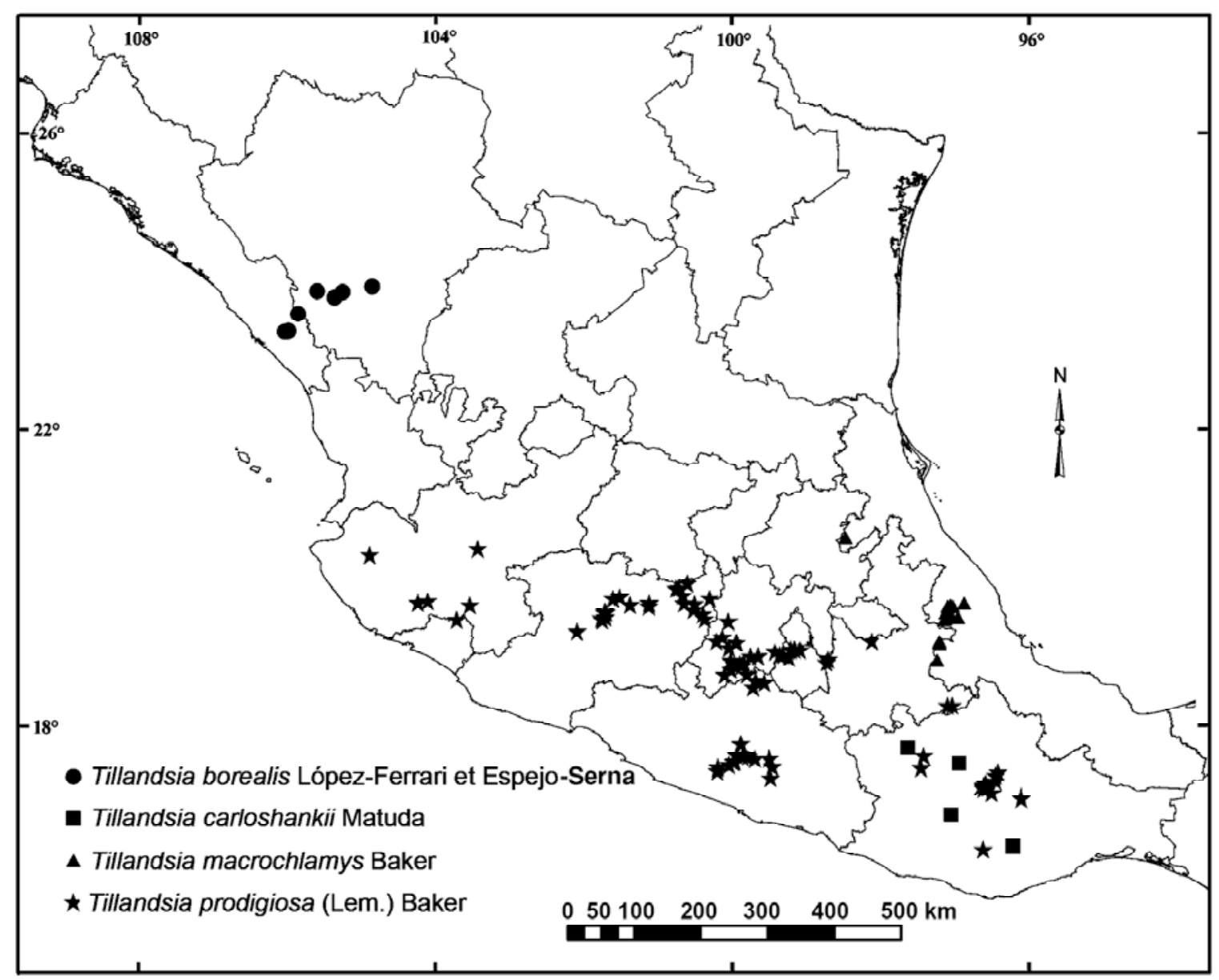

Figura 3. Distribución conocida de Tillandsia carloshankii Matuda (₫), Tillandsia macrochlamys Baker (ム), Tillandsia prodigiosa (Lem.) Baker ( $\star$ y Tillandsia borealis López-Ferrari et Espejo-Serna $(\bullet)$.

from Central Mexico. Acta Botanica Mexicana 78:85-95.

Gardner C.S. 1986. Preliminary classification of Tillandsia based on floral characters. Selbyana 9:130-146.

Grant J.R. 1995a. Werauhia, a new ge nus. Tropische und Subtropische Pflanzenwelt 91:16-52.

Grant J.R. 1995b. Addendum to "The resurrection of Alcantarea and Werauhia, a new genus" (Bromeliaceae: Tillandsioideae). Phytologia 78:119-123.

Holmgren P.K., Holmgren N.H. y Barnett L.C. 1990. Index Herbariorum. Part I: The Herbaria of the World (Regnum Vegetabile, vol. 120). New York Botanical Garden, Nueva York. López-Ferrari A.R., Espejo-Serna A. y Blanco Fernández de Caleya P. 2006. Circunscripción de Tillandsia chaetophylla Mez y descripción de Tillandsia sessemocinoi (Bromeliaceae: Tillandsioideae). Acta Botanica Mexicana 76:77-88.
Mez C. 1896. Bromeliaceae. Monographie Phanerogamarum 9:1990.

Smith L.B. y Downs R.J. 1977. Tillandsioideae (Bromeliaceae). Flora Neotropica Monograph 14:1-1492.

Spencer M.A. y Smith L.B. 1993. Racinaea, a new genus of Bromeliaceae (Tillandsioideae). Phytologia 74:151-160.

Terry R.G., Brown G.K. y Olmstead R.G. 1997. Phylogenetic relationships in subfamily Tillandsioideae (Bromeliaceae), using $n h d F$ sequences. Sytematic Botany 22:333-345.

Till W. y Barfuss M.H.J. 2006. Progress towards a new classification of Tillandsioideae. Journal of the Bromeliad Society 56:253-259.

Recibido: 23 de enero de 2007

Versión corregida: 5 de marzo de 2007

Aceptado: 6 de marzo de 2007 
TILLANDSIA BOREALIS, NUEVA ESPECIE DEL NORTE DE MÉXICO

Apéndice 1. Lista de exsiccata revisados. Los acrónimos de los herbarios según Holmgren et al. (1990).

Tillandsia carloshankii Matuda. Figura $1 \mathrm{a}$.

Oaxaca: Distrito de Cuicatlán, Municipio de Santiago Nacaltepec, $3 \mathrm{~km}$ al $\mathrm{N}$ de Cieneguilla, rumbo a Tehuacán, $17^{\circ} 29^{\prime} 24^{\prime \prime}$ N, 9656'21" O, 1,220 m, 4-09-2002, A. Espejo-Serna, A.R. López-Ferrari, J. Ceja y A. Mendoza R. 6505 [UAMIZ(4)]; Distrito de Yautepec, Municipio de San Carlos Yautepec, Sierra de Santiago Lachivía, "Lacheguía", 28-101972, E. Matuda y colaboradores 38514 (Holotipo: MEXU, Isotipo: CODAGEM).

Tillandsia macrochlamys Baker. Figura $1 \mathrm{~b}$.

Veracruz: Municipio de Acajete, camino La Joya-Las Lajas, M. Cházaro y P. Sánchez 2090 (MEXU); 5 km al O de La Joya, A. Gómez-Pompa 1433 (MEXU); La Joya, between Perote and Xalapa, on hill $500 \mathrm{~m} \mathrm{~N} \mathrm{of} \mathrm{the} \mathrm{road,} \mathrm{P.} \mathrm{Hietz} 215$ (XAL); al N de La Joya, F. Ventura 198 [ENCB(2), IEB(2)]; Municipio de Calcahualco, J. Ceja et al. 434 [UAMIZ(3)]; San Miguel Tlacotiopa, J.L. Martínez 94 (XAL); 3 km al O de Rincón Atotonilco, J.L. Martínez y A. Hernández 1308 (XAL); barranca de San Miguel Tlacotiopa, P. Tenorio 15463 (MEXU); Municipio de Coatepec, abajo de Mesa de los Laureles, M. Cházaro y P. Hernández de Cházaro 3991 (XAL); Cofre de Perote, entre Mesa de los Laureles y Tierra Grande, M. Cházaro y L.E. Castillo 6790 (XAL); Municipio de Huayacocotla, cerca de Huayacocotla, R. Hernández y R. Cedillo 1034 (IEB); Municipio de Ixhuacán, barranca La Funda, cerca de Los Laureles, al S del Cofre de Perote, H. Narave y F. Vázquez 1049 [MEXU, XAL(2)]; Municipio de Maltrata, Xúchil, base de I'Orizaba, 2,700 m, I.1908, reçue en fl. de Morelia, 1909, fr. Arsène 1922 (P); Municipio de Naolinco, cerca de Naolinco, Q.D. O'Hare s. n. (XAL); Municipio de Perote, por la brecha que va a Tonalaco, en las faldas del Cofre de Perote, G. Castillo et al. 1984 (XAL); terre froide du Cofre de Perote. Xalapa "Jalapa", L. Hahn 589 [Holotipo: P(2), isotipo: K]; Municipio de Las Vigas, El Volcancillo, $R$. Ortega 580 (XAL); Las Lajas, al SE de Las Lajas, P. Zamora y G. Castillo 2190 (IEB, XAL); Municipio de Xico, entre Corral de Rajas (Ingenio del Rosario) y Buenavista, Cofre de Perote, M. Cházaro y H. Oliva 4301 (XAL); abajo del Ingenio del Rosario rumbo a Buenavista, M. Cházaro et al. 5844 (IBUG); 500 m antes de Corral de Rajas, J. Martínez-García 144 (XAL); ingenio El Rosario, H. Narave et al. 287 (XAL).

Tillandsia prodigiosa Baker. Figura 1c.

Colima: Municipio de Comala, $3 \mathrm{~km}$ después del crucero camino a San Antonio, M. Huerta, S. Guerrero, A. Ruiz et al. 236 (IBUG, IEB, XAL). Guerrero: municipio de Atoyac de Álvarez, Vic. of Nueva Dehli, K. Ehlers y R. Ehlers EM901001 (SEL); en El Ranchito, 13 km al NE de El Paraíso, J.C. Soto N. y S. Aureoles C. 7867 (MEXU); Municipio de Chilpancingo de los Bravo, Rincón de la Vía, H. Kruse s. n. sub E. Matuda 38632 (LL, MEXU); Sierra de Tezcaltitemi, Mazatlán, H. Kruse 3103 (MEXU); Sierra Madre, E. Langlassé 1026 [GH, P(2)]; Sierra de Tezcaltitemi, Mazatlán, E. Matuda 3103 [MO (3)]; 1 mile west Omiltemi, T.W. McCorcle y C.M. Rowell, Jr 3451 (MICH); Municipio de General Heliodoro Castillo, Tres Caminos, $13.5 \mathrm{~km}$ al SO rumbo a El jilguero, J. Calónico S. 8432 (FCME); 5.03 km al N de Verde Rico, J. Calónico S. 12522 (FCME); $5.59 \mathrm{~km}$ al N de Verde Rico, J. Calónico S. 12601 (FCME); $8 \mathrm{~km}$ al SO de Puerto El Jilguero, camino a Puerto del Gallo, F. Lorea 3938 (FCME); Yerba Buena, sierra costal de Guerrero, E. Matuda 38654 (MEXU); Municipio de Ixcateopan de Cuauhtémoc, San Miguel, 2.5 km al N, camino Zumpango - Taxco, R. Cruz Durán 2167 (FCME); Municipio de Leonardo Bravo, 9 km de Tres Caminos rumbo a Yextla, J. Calónico S. 3549 (FCME); aproximadamente a $2.5 \mathrm{~km}$ adelante de Carrizal de Bravos, rumbo a Atoyac, A.R. López-Ferrari, A. Espejo-Serna y T. Chehaibar 362 [UAMIZ (6)]; $5 \mathrm{~km}$ al SE de El Carrizal de los Bravos, camino Filo de Caballo - Chichihualco, E. Martínez S., T.P. Ramamoorthy y J. Miller 3319 (ENCB, MEXU); 9 km al SO de Filo de Caballo, carretera a Puerto del Gallo, J.C. Soto N. y F. Solórzano G. 12689 [MEXU (2), UAMIZ]; Municipio de Taxco de Alarcón, 6 km al NO de Taxco de Alarcón, J. Calónico S. 3698 (FCME); $10 \mathrm{~km}$ al ONO de Taxco, sobre el camino a Tenería, J. Rzedowski 25242 a [ENCB (3)]; sin municipio indicado, along the road from Milpillas to Nueva Dehli, H. y L. Hromadnik 14068 [UAMIZ, WU(2)]; Vientos Fríos - Yerba Buena, 1,800 m, R. Ehlers y K. Ehlers EM900906 [WU (7)]. Jalisco: Municipio de Autlán de Navarro, Brecha Ahuacapán Las Joyas de Manantlán, entre Corralitos y Cuatro Caminos, R. Ramírez D., R. González Tamayo y F.J. Santana M. 1247 [IBUG (2)]; Municipio de Cuautitlán de García Barragán, La Ventana, Sierra de Manantlán, L.M.V. de Puga 13772 (IBUG); Municipio de Talpa de Allende, Sierra de Cuale, southwest of Talpa de Allende, southwest of the prominent peak called Piedra Rajada, R. McVaugh 14351 [MICH (2)]; Municipio de Tlajomulco de Zuñiga, Cerro Viejo, cara norte, barranca Las Crucecitas, al suroeste de San Miguel Cuyutlán, J.A. Machuca N. y M. Cházaro B. 6626 (IEB, MEXU, TEX, XAL); Municipio de Zapotlán el Grande, 2-3 km después de la carretera, por la brecha que va hacia Las Víboras (microondas), desde el Puerto del Floripondio, M. Huerta M., M. Cházaro B., E. Lomelí y R. Patiño 42 [IEB (2), XAL]. Michoacán: Municipio de Erongarícuaro, al E de Charahuén, H. Díaz Barriga 5272a [IBUG (2), IEB (2)]; Opongio, J.M. Escobedo 674 [ENCB (2), IEB (3), MEXU]; Municipio de Hidalgo, 8-10 miles north of west de Ciudad Hidalgo and a few miles north of village of San Pedro Jácuaro "Aguaro", R. McVaugh 9996 (MEXU, US); Municipio de Morelia, Aguazarca, C. Medina G. 928 [IEB (2)]; 2 km al sur de San José de las Torres, J. Rzedowski 42880 (IEB); ladera noreste del Cerro del Águila, S. Zamudio R. 6035 [IEB (2), MEXU (2)]; Municipio de Pátzcuaro, ca. 2 km al N de Ajuno, cerro La Taza, M.E. 
ANA Rosa LóPEZ-FERRARI y AdOLFO ESPEJO-SERNA

Molina 339 [IEB (2), UAMIZ]; Municipio de Quiroga, Cerro del Tziráte, J.M. Escobedo 1296 [IEB (2)]; 24 km al E de Zacapu, por la carretera a Quiroga, S.D. Koch y M. González L. 8687 (CIIDIR, ENCB); Cuesta de Aratzipo, km 372 on highway between Quiroga and Zacapu, H.E. Moore Jr. y C.E. Wood Jr. 4044 (AA); Municipio de Salvador Escalante, camino al cerro San Miguel, cerca de Zirahuén, H. Díaz Barriga 1760 [IEB (3)]; 1 km de Copándaro, E. Pérez Cálix 303 [IEB (2)]; Agua Verde, en el extremo SO del lago Zirahuén, S. Zamudio R. y D. Tejero D. 11863 (IEB); Municipio de Senguio, Peña Blanca, ejido San Francisco de los Reyes, I. García R. y H. de García 3601 [IEB (2), UAMIZ (2)]; Municipio de Tuxpan, La Joya Verde, al oeste de Las Caleras, $12 \mathrm{~km}$ al oeste de Tuxpan, M. Torres y M. Ramírez 2265 (IEB); El Aguacate, $8.5 \mathrm{~km}$ al SO de Malacote, camino a Agostitlán, R. Torres y M. Ramírez 13491 [IEB (2)]; Municipio de Uruapan, west facing slopes of cerro Carboneras above the río Cupatitzio, ca. $22 \mathrm{~km}$ south of Uruapan, R.M. King $y$ T.R. Soderstrom 4896 (US); Municipio de Zinapécuaro, cerro El Mozo, al SO de Ucareo, E. Carranza G. 4092 [IEB, MEXU, UAMIZ (2)]; $500 \mathrm{~m}$ al oeste del balneario Las Adjuntas, M.J. Jasso 843 [ENCB (2), IEB (2), MEXU (2), XAL]; Cerro de la Cruz, 2 km al norte de Ucareo, S. Zamudio R. 3829 (CIIDIR, ENCB, IBUG, IEB, MEXU); Municipio de Zitácuaro, $5 \mathrm{~km}$ al NE de San Felipe de los Alzati, J.C. Soto N., A. Román de Soto y F. Soto R. 6501 (MEXU, MO); Coatepec de Morelos, $4 \mathrm{~km}$ al suroeste de Zitácuaro, J.C. Soto N. y S. Aureoles C. 7337 (IEB). Morelos: Municipio de Cuernavaca, 2 km adelante de Tlatempa, rumbo a Buenavista del Monte, J. Ceja, A. Espejo-Serna, A.R. López-Ferrari y A. Mendoza R. 807 [CICY (2), IEB (3), UAMIZ (2)]; Municipio de Tepoztlán, pedregal del derrame del Chichinautzin, km 65 de la carretera de cuota México - Cuernavaca, A. Espejo-Serna, A.R. López-Ferrari, J. Ceja y A. Mendoza R. 6029 [CICY (3), IEB (4), UAMIZ (3)]; Sierra de Tepoztlán, cerros al E de San Juan Tlacotenco, A.R. López-Ferrari, A. Espejo-Serna, J. GarcíaCruz y R. Jiménez M. 2371 [IEB (2), UAMIZ (5)]; derrame del Chichinautzin, S. Zamudio R. s. n. (FCME); Municipio de Tetela del Volcán, barranca de Amatzinac, A.R. López-Ferrari, A. Espejo-Serna, J. García-Cruz y R. Jiménez M. 2432 [UAMIZ (2)]; ca. $5 \mathrm{~km}$ sobre la desviación a la cascada El Salto, a partir del camino Tetela del Volcán - Hueyapan, J. Santana C., L. Pacheco, Y. Sandoval, E. Callejas G. y A. Valdés R. 384b [UAMIZ (4)]. Estado de México: Municipio de Almoloya de Alquisiras, aproximadamente $3 \mathrm{~km}$ al O de Plan de Vigas, aproximadamente $2 \mathrm{~km}$ al E de El Potrero, $A$. Espejo-Serna, M. Flores C., G. Barroso Ch. y G. Calzada 3833A [UAMIZ (8)]; El Puerto, aproximadamente $7 \mathrm{~km}$ al SE de Almoloya de Alquisiras, M. Flores C. y F. Riveros 790 [UAMIZ (4)]; Las Iglesias, SE de Almoloya de Alquisiras, M. Flores C. 920 [UAMIZ (3)]; Municipio de Amatepec, 0.5 km después de la desviación a La Goleta, sobre la carretera Amatepec - Sultepec, M. Flores C., R. Grether, A. Martínez B. y S. Camargo R. 705 [UAMIZ (6)]; Municipio de Coatepec Harinas, $3 \mathrm{~km}$ al noreste de Coatepec Harinas, sobre el camino a Agua Amarga, J. Rzedowski 30345 [ENCB (2), IEB (2)]; Municipio de Ocuilan, Ahuacatitlán, $10 \mathrm{~km}$ después de Ahuatenco, carretera Cuernavaca - Ocuilan, M. Flores C. y F. Riveros 978 [UAMIZ (4)]; Santa Mónica Ocuilan, M.E. Huidobro S. 164 (MEXU); Municipio de Otzoloapan, Cerro de Pinal, Otzoloapan, E. Matuda y colaboradores 31763 [MEXU (2), US]; Municipio de Sultepec, entre Sultepec y Amatepec, E. Matuda y colaboradores 30080 (MEXU, US); $5 \mathrm{~km}$ al SO de Sultepec, sobre el camino a Amatepec, J. Rzedowski 36050 [ENCB (2), IEB (2)]; Municipio de Tejupilco, district Temascaltepec. Pantoja, G.B. Hinton et al. 7437 [GH (2)]; Municipio de Temascaltepec, Temascaltepec, H. Brailovsky s. n. (MEXU); 2 km después de La Comunidad, carretera Toluca - Temascaltepec, M. Flores C. y F. Riveros 676 [UAMIZ (2)]; 5 km después de La Comunidad y $500 \mathrm{~m}$ antes de Cieneguillas, carretera Toluca - Temascaltepec, M. Flores C. y F. Riveros 683 (UAMIZ); Temascaltepec, E. Matuda y colaboradores 27826 [MEXU (2)], E. Matuda y colaboradores 27910 (MEXU), E. Matuda s. n. [MEXU (2)]; Municipio de Valle de Bravo, Valle de Bravo, E. Matuda y colaboradores 27754 [MEXU (2)]; Municipio de Villa de Allende, 12 km después de Villa Victoria, carretera El Oro - Valle de Bravo, M. Flores C. y F. Riveros 957 (UAMIZ); Municipio de Villa Guerrero, Carretera Tenancingo - Ixtapan de la Sal, $5 \mathrm{~km}$ al O de Villa Guerrero (Cerca del puente Santa María), J. Arellano, K. Baker, S. Montes y C. Mapes 419 (ENCB, XAL); km 50 de la Carretera Tenancingo - Ixtapan de la Sal, puente de Santa María, $5 \mathrm{~km}$ al O de Villa Guerrero, K. Baker, J. Arellano, C. Mapes y S. Montes 19 [IEB (2) MEXU, MICH]; Municipio de Zacualpan, Cerro Tres Coronas, A. Espejo-Serna, A.R. López-Ferrari y M. Flores C. 4723 [UAMIZ (2)]; sin Mpio. indicado, district Temascaltepec. above Mina Rincón, H.E. Moore Jr. 2501 (GH). Oaxaca: distrito de Ixtlán, Municipio de Capulalpam de Méndez, vicinity of Hwy 175 from Valle Nacional to Oaxaca, vicinity of Ixtlán de Juárez, along road between Ixtlán de Juárez and Calpulalpan, ca. 1 mile northwest of Calpulalpan near bridge, vicinity km 8, T.B. Croat y D.P. Hannon 65656 (MO); Capulalpam, noroeste del río Molinos, tierra caliente, S. Figueroa B. y Y. Guzmán R. 198 (CHAP); Municipio de Ixtlán de Juárez, near Loma Grande, ca. 26 km north of Oaxaca on hwy 175 , D.H. Norris y D.J. Taranto 16661 (MEXU); Municipio de Santa Catarina Ixtepeji, $30 \mathrm{~km}$ al S de Ixtlán de Juárez, $R$. Cedillo T. y D.H. Lorence 637 (MEXU, MO); vicinity of the pueblo Latuvi near Santa Catarina Lachatao, ca. $30 \mathrm{~km} \mathrm{NE}$ of Oaxaca (10 km E of S. C. Ixtepeji, 15 km S of Ixtlán), H.H. Iltis 27148 (MEXU); carretera Oaxaca Tuxtepec, 2 km al $\mathrm{N}$ de El Punto, S.D. Koch y M. González L. 86113 (ENCB); alrededores de El Punto, A.R. López-Ferrari, A. Espejo-Serna, J. Ceja, A. Mendoza R. y G. Carnevali 3069 [UAMIZ (6)]; carretera Oaxaca a Ixtlán, cerca El Punto, F. Miranda 8389 [MEXU (2)]; distrito de Miahuatlán, Municipio de Miahuatlán de Porfirio Díaz, km 100 de la carretera Oaxaca - Puerto Ángel, Solano S.C. y Vara M.A. 243 (MEXU); distrito Mixe, Municipio de Totontepec Villa de Morelos, 37 km después de Santa María Yacochi y ca. 14 después de Tamazulapan del Espíritu Santo, rumbo a Mitla, A. Espejo-Serna, A.R. LópezFerrari, J. Ceja y A. Mendoza R. 6676 [UAMIZ (4)]; distrito Teposcolula, Municipio de San Juan Teposcolula, cerro 
Yucudaá, torre de microondas, A. García-Mendoza y J. Reyes 4962 (MEXU); Municipio de San Vicente Nuñu, alrededores de Anama, $3 \mathrm{~km}$ al S de San Vicente Nuñu, A. García-Mendoza y J. Reyes 5211 (MEXU); distrito de Tlacolula, Municipio de San Pablo Villa de Mitla, eastern slope between Mitla and Cuesta, W.R. Ernst 2466 [MEXU (3), MICH, US (2)]; Municipio de Teotitlán del Valle, brecha de Teotitlán del Valle a Benito Juárez, M. Cházaro B., M. Kimnach, $R$. Dorsch y M. Negrete 6831 (MEXU, XAL); sin distrito ni municipio indicados, sin localidad indicada, Fl. in cult., P. Koide s. n. (F) (from Sierra Juárez), T. MacDougall s. n. (MO). Puebla: Municipio de Coxcatlán, above Coxcatlán between Pala and the top of cerro Chichiltepec, C.E. Smith Jr., F.A. Peterson y N. Tejeda 3883 (US); Municipio de Coyomeapan, aproximadamente $30 \mathrm{~km}$ al NE de Coxcatlán, rumbo a Coyomeapa, a partir del Camino Tehuacán - Teotitlán del Camino, J. Santana C., A. Martínez B. y S. Camargo R. 454 [UAMIZ (2)]; Municipio de Puebla, Malinche, près Puebla, Bro. Nicolás s. n. $[\mathrm{P}(2)]$. 